\title{
Necrotising otitis externa in the immunocompetent patient: case series
}

\author{
S UNADKAT, T KANZARA, G WATTERS \\ Department of Otolaryngology, Southend University Hospitals NHS Trust, UK
}

\begin{abstract}
Background: Necrotising otitis externa can be a devastating form of otitis externa. It typically tends to affect patients who are immunocompromised or diabetic. To date, there is very little in the literature about necrotising otitis externa in the immunocompetent patient population.

Case reports: The present paper discusses both the clinical and radiological findings in three cases of necrotising otitis externa in an immunocompetent patient cohort. The common factor among all three patients was their advanced age.

Conclusion: Diagnosing necrotising otitis externa can be challenging because of the potentially non-specific symptoms and the absence of early radiological signs, particularly if patients are neither immunocompromised nor diabetic. Elderly patients should be considered in the same light as immunocompromised and diabetic patients in the context of necrotising otitis externa.
\end{abstract}

Key words: Adult; Aged; Aged, 80 And Over; Anti-Infective Agents; Therapeutic Uses; Diabetes Complications; Diagnostic Imaging; Disease Progression; Drug Therapy, Combination; Female; Gallium Radioisotopes; Humans; Immunocompetence; Immunocompromised Host; Male; Otitis Externa; Diagnosis; Etiology; Therapy; Pseudomonas Infections; Retrospective Studies; Technetium

\section{Introduction}

Necrotising (or malignant) otitis externa is a well-recognised and potentially life-threatening form of otitis externa, which typically occurs in patients who are immunocompromised, particularly those with diabetes. Historically, it has not been identified in immunocompetent individuals.

This paper reports three cases of necrotising otitis externa in patients who had no underlying medical condition causing them to be immunocompromised, though a common factor in all three cases was their advanced age.

\section{Case reports}

Case one

An 87-year-old man presented in April 2011 with a 6-week history of left-sided otalgia and otorrhoea. Examination revealed polypoidal tissue in the ear canal. This tissue was biopsied and histology showed inflammatory granulation tissue. Audiometry showed a moderate-to-severe mixed hearing loss in the left ear. An ear swab grew pseudomonas.

The patient was treated with both oral and topical ciprofloxacin for two weeks. However, the otalgia intensified, and in May he developed a left facial nerve palsy (House-Brackmann grade V). He was admitted for pain control and intravenous Tazocin ${ }^{\circledR}$. The findings of blood tests, including serum glucose, were normal, apart from raised white blood cell count and C-reactive protein (CRP), consistent with infection. At this time, a computed tomography (CT) scan of the temporal bones showed no abnormality. The otalgia improved, but his facial nerve palsy progressed to House-Brackmann grade VI. An isotope bone scan showed uptake in the left temporal bone consistent with osteomyelitis, and so the patient was readmitted for intravenous Tazocin. He was discharged after two weeks, and, on the advice of the microbiology department, given a further two weeks of oral ciprofloxacin and flucloxacillin. This resolved his pain, but not his facial nerve palsy.

In August 2011, the pain returned, radiating to the throat, with a global deterioration of the patient's health. Evaluation of inflammatory markers showed a rising CRP level. A repeat CT scan (Figure 1) and magnetic resonance imaging (MRI) scan in September 2011 (Figure 2) showed a mass involving the skull base, with extensive destruction of the temporal bone and extension into the retropharyngeal space. Clinically, the patient responded well to intravenous antibiotics, although he had now developed a left vagal nerve palsy, causing choking and difficulty swallowing. The ear canal had healed and the tympanic membrane looked normal, so a neoplastic process was considered. However, it was felt that the patient was unsuitable for any form of radical treatment for a malignancy and obtaining a further biopsy would be a relatively major procedure.

The patient continued to improve clinically with intravenous antibiotics, and he was discharged on oral ciprofloxacin and flucloxacillin for two months. A further MRI scan (Figure 3) performed three months after discharge from hospital showed a reduction in the size of the mass evident on the first scan.

On review in April 2012, the patient's swallowing had returned to normal, and flexible laryngoscopy confirmed 


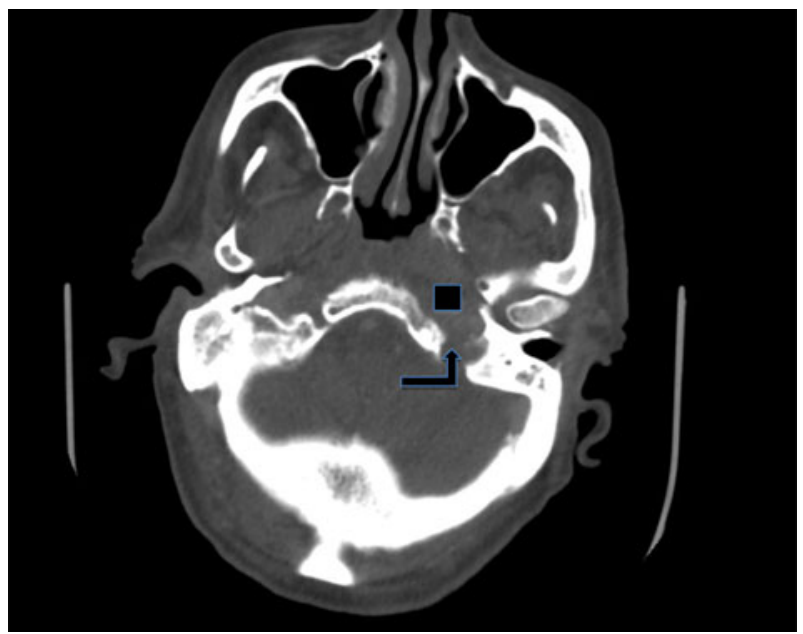

FIG. 1

Axial computed tomography scan of the temporal bone in case one, demonstrating a soft tissue density lesion arising from the skull base and extending into the retropharynx (square), with temporal bone destruction (arrow)

partial recovery of vagal nerve function. An MRI scan conducted in June 2012 showed almost complete resolution of the retropharyngeal and skull base mass. However, there was no recovery of facial nerve function. He was referred on to the plastic surgeons, who undertook static facelift surgery coupled with a tarsorrhaphy in 2014.

As of February 2017, although clinically well, the patient remains under the care of the physicians for dementia assessment.

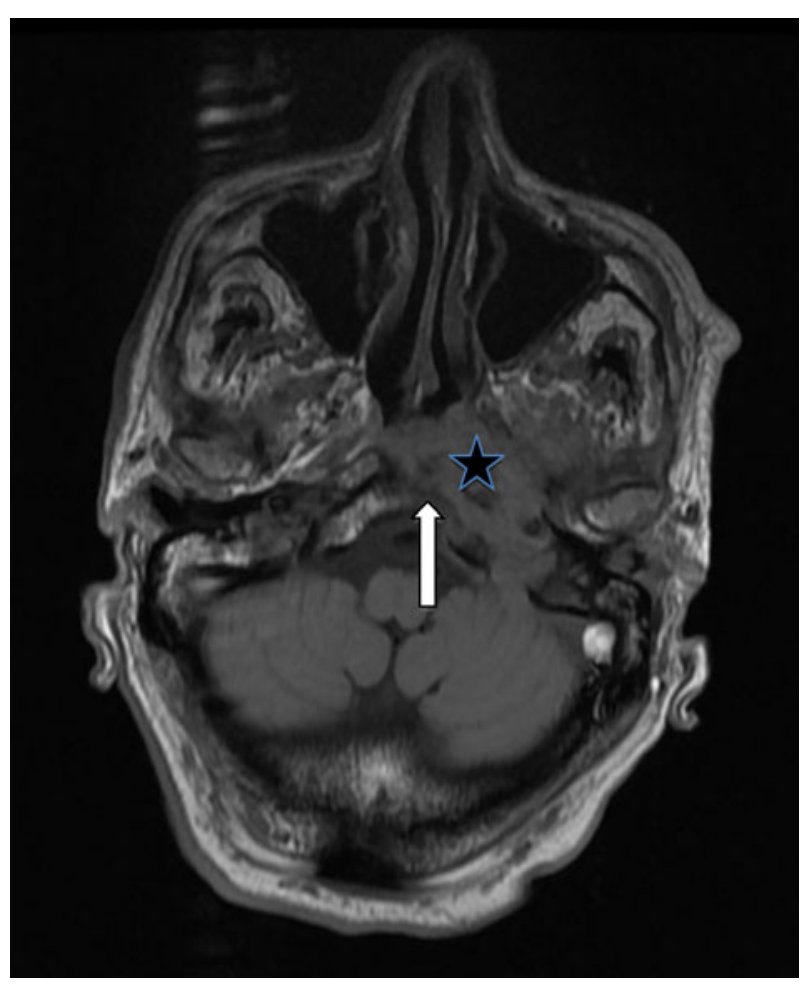

FIG. 2

Axial T1-weighted magnetic resonance imaging scan with contrast, of case one, demonstrating a left-sided soft tissue mass arising from the skull base and extending into the nasopharynx (star). There is erosion of the clivus, denoted by the abnormal marrow signal (arrow).

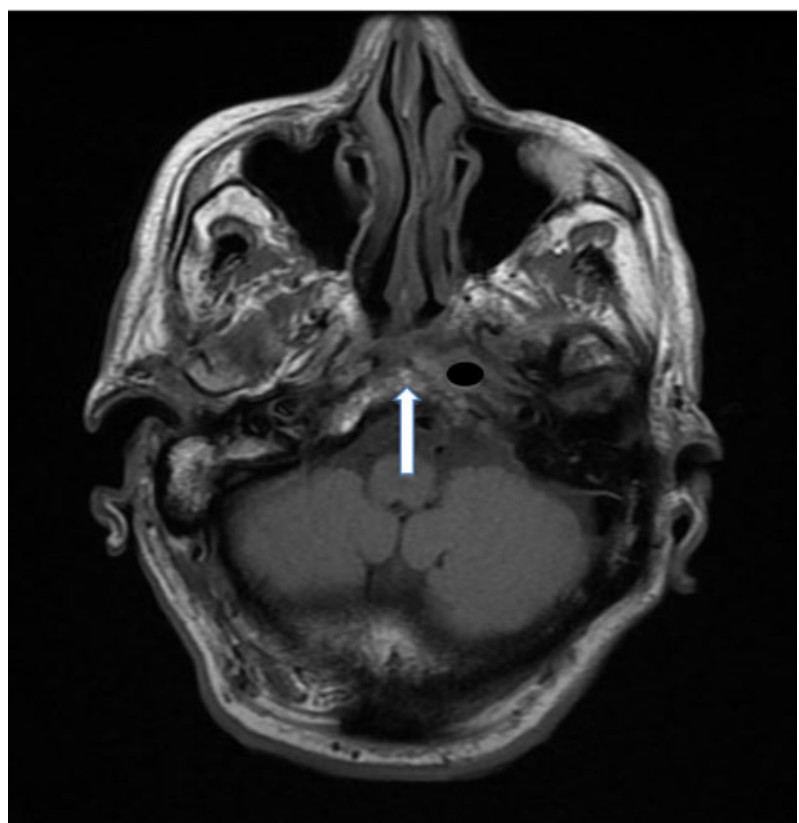

FIG. 3

Axial magnetic resonance imaging scan with contrast, of case one, performed three months after discharge from hospital, demonstrating incomplete resolution of the soft tissue mass (oval) and the return of normal marrow signalling in the clivus (arrow).

\section{Case two}

A 93-year-old man, with a history of atrial fibrillation, hypertension and hypothyroidism, presented to the ENT clinic in August 2013 with left-sided pain, hearing loss and purulent otorrhoea, in keeping with otitis externa. Initial examination demonstrated granulation tissue in the floor of the ear canal and facial nerve palsy (House-Brackmann grade II). He had no significant underlying medical condition causing him to be immunocompromised, and findings of blood tests, including serum glucose, were normal. He was admitted for intravenous antibiotics (Tazocin) and intensive topical treatment to the ear canal, including daily micro-suctioning. Pseudomonas and candida were isolated from the ear swab. An audiogram showed a moderate-to-severe mixed hearing loss. An initial CT scan of the temporal bones showed no evidence of osteomyelitis.

Having completed a 15-day course of intravenous ceftazidime, the patient was discharged on oral ciprofloxacin for 4 weeks. Although his pain improved, the facial nerve palsy progressed to House-Brackmann grade VI. A repeat CT scan conducted in September 2013 (Figure 4) now showed

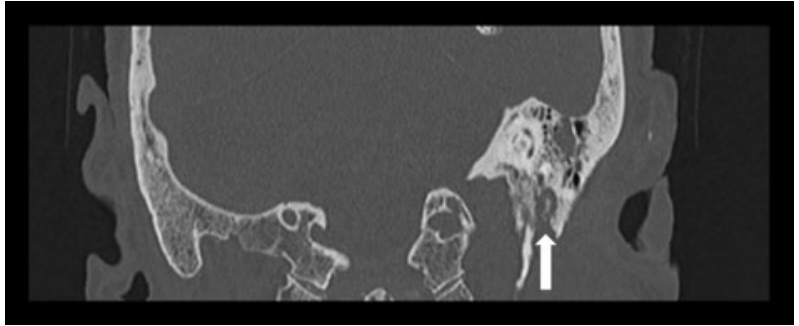

FIG. 4

Coronal high-resolution computed tomography scan of the temporal bones in case two, demonstrating bone destruction of the external auditory canal and stylomastoid foramen (arrow). 
destruction in the bone of the ear canal floor. An MRI scan showed an inflammatory mass in the left temporal bone, with apparent invasion of the stylomastoid foramen. In view of the radiology findings, he was readmitted for further intravenous antibiotic treatment (ceftazidime) and a biopsy of the ear canal, which confirmed inflammatory granulation tissue. His pain resolved completely and he was discharged with six weeks of oral ciprofloxacin.

Upon review in October 2014, there had been no recovery of facial nerve function. In December 2014, the patient underwent a static facelift and tarsorrhaphy under the plastic surgeons. As of April 2017, he remains clinically well.

\section{Case three}

A 93-year-old woman presented to the emergency department in January 2014 with a severe headache and a hoarse voice. Over the preceding three months, she had been seen on nine separate occasions with chronic otitis externa, which was treated with a combination of oral and topical antibiotics. She was not immunocompromised or diabetic, as confirmed via blood tests.

Examination revealed a right vocal fold palsy, but facial nerve function was normal. Otoscopy showed a right ear canal polyp occluding the canal, but no active otorrhoea. Clinically, the patient had otitis externa. She was admitted for intravenous co-amoxiclav and was also started on topical Gentisone ${ }^{\circledR} \mathrm{HC}$.

A CT scan of the temporal bones was unimpressive, but revealed bony destruction of: the right mastoid air cells, the petrous part of the temporal bone and the occipital bone. No intracranial abscesses were seen. Based on microbiology advice, treatment was changed to intravenous meropenem and topical ciprofloxacin.

Antimicrobial therapy continued for a total of three weeks, in hospital, with nasogastric tube feeding. By discharge, the patient's swallowing problems and vagal nerve palsy had resolved. Oral ciprofloxacin was prescribed for a further three weeks after discharge. As of March 2017, she remains asymptomatic.

\section{Discussion}

Necrotising otitis externa is a well-recognised form of otitis externa in the diabetic and immunocompromised patient population. The most commonly implicated pathogenic organism is Pseudomonas aeruginosa, and this correlates precisely with our case series. It is hypothesised that the pathogenesis of the condition relates to microangiopathy, hypoperfusion and diminished host resistance due to diabetes. ${ }^{1,2}$ The infection is thought to spread from the external auditory canal, to the temporal bone and skull base, via the fissure of Santorini and the osseocartilaginous junction.

To date, there have been very few reports of this severe form of otitis externa in immunocompetent individuals. Su et al. reported one such case, but it was complicated by preceding mastoid surgery, ${ }^{3}$ and so may not represent a true case of temporal bone osteomyelitis arising from otitis externa. In the case report by Cunningham et al., the isolated organism was aspergillus and not pseudomonas. ${ }^{4}$ Extensive review of the English-language literature failed to identify any other cases of necrotising otitis externa due to pseudomonas affecting immunocompetent patients.

In all three of our cases, the diagnosis of necrotising otitis externa was not considered at the time of initial presentation, as none of the patients were diabetic and none had any other medical conditions associated with immune system impairment. The only common factor in our series appears to be advanced age. It is widely accepted that ageing diminishes the host's ability to mount an effective immune response. ${ }^{5}$ Factors such as age-related dysregulation of the cytokine milieu, defective haematopoiesis and chronic thymic involution have all been implicated in age-related immunoscenescence. ${ }^{5-7}$

Interestingly, perhaps because of the perceived lack of association of the condition to the elderly and subsequent delayed diagnoses, all of the patients developed cranial nerve palsies: a facial nerve palsy (House-Brackmann grade VII) in cases one and two, and a vagal nerve palsy (grade X) in cases one and three. Although the vagal nerve palsies recovered, the facial nerve palsies did not. According to Illing and Olaleye, ${ }^{2}$ the mortality rate rises from 33 per cent to 80 per cent with cranial nerve involvement, although this has been disputed in other studies which suggest a comparable survival rate in both groups. ${ }^{8}$

Another interesting point in cases one and two was that initial CT scans were reported as normal, and bone destruction only became apparent later once cranial nerve involvement had progressed. This aptly illustrates the limitations of reliance on CT scans alone, and underlines the importance of using $\mathrm{CT}$ in combination with other imaging modalities like radioisotope scanning and MRI.

Whilst CT scans can be useful in determining the location and extent of disease, particularly bony erosion, pathology tends to show late on CT because at least a third of the temporal bone must be eroded before pathology becomes obvious radiologically. As a corollary, CT is of limited utility in monitoring the treatment of infection because bone remineralisation continues long after resolution. ${ }^{9}$

Magnetic resonance imaging is useful for illustrating soft tissue changes like dural enhancement and involvement of medullary bone spaces, which manifest as abnormal marrow signalling. However, as with $\mathrm{CT}$, these changes can persist long after infection resolution, making MRI unsuitable for confirming resolution. ${ }^{10}$

- Necrotising otitis externa is potentially life-threatening and carries significant morbidity associated with cranial nerve involvement

- Late diagnosis is commonplace

- Radiological findings may not reflect the true clinical picture

- Necrotising otitis externa can also be found in immunocompetent patients, particularly in those of advancing age

- Elderly patients should be considered in the same light as immunocompromised individuals in the context of necrotising otitis externa

- Treatment must be initiated on clinical suspicion, even if no radiological findings, and later repeated, potentially with alternative imaging modalities

Radioisotope scans are useful adjuncts to both CT and MRI; however, they are not widely available in day-to-day practice. Technetium-99 m radioisotope methylene diphosphonate scintigraphy offers a sensitivity of 100 per cent in diagnosing necrotising otitis externa, and allows earlier diagnosis of skull base osteomyelitis than $\mathrm{CT}$ alone. ${ }^{11}$ Unfortunately, 
technetium-99 m (methylene diphosphonate), though useful, cannot discriminate between infective and non-infective processes. $^{10,11}$ This mandates the exclusion of a neoplastic process before confirming a diagnosis of necrotising otitis externa. Furthermore, technetium-99 m methylene diphosphonate remains positive for as long as bone repair continues, rendering it unreliable in the follow up of patients with necrotising otitis externa. $^{11}$

Gallium citrate $\mathrm{Ga} 67$ radioisotope scanning, if available, is particularly useful for diagnosis and follow up. This is because the isotope binds to bacteria, and is absorbed by both macrophages and other cell components of the reticuloendothelial system, making the detection of infection more predictable: normal uptake indicates resolution of infection. ${ }^{12,13}$

In conclusion, whilst traditional teaching has centred on considering the possibility of necrotising otitis externa in diabetics and patients who are otherwise immunocompromised, we propose that a third group, the very elderly patient population, ought to be considered in the same light. Given that each of our patients developed cranial nerve palsies, doctors treating the elderly for otitis externa need to ensure that these patients are closely monitored. Furthermore, clinicians should have a lower threshold for initiating aggressive medical treatment with intravenous antibiotics. Our series also shows that early imaging with CT scans may be misleading, and imaging should be repeated if there is a suggestion of clinical progression, particularly cranial nerve involvement.

\section{Acknowledgement}

We thank the medical records department at Southend University Hospital for its help in obtaining patient notes.

\section{References}

1 Singh A, Al Khabori M, Hyder MJ. Skull base osteomyelitis: diagnosis and therapeutic challenges in atypical presentation. Otolaryngol Head Neck Surg 2005;133:121-5

2 Illing E, Olaleye O. Malignant otitis externa: a review of aetiology, presentation, investigations and current management strategies. Webmed Central Otorhinolaryngology 2011;2: WMC001725

$3 \mathrm{Su}$ N, Syed I, Garth R. Skull based osteomyelitis due to postsurgery malignant otitis externa presenting as stroke. BMJ Case Rep 2011;bcr0220113908

4 Cunningham M, Yu VL, Turner J, Curtin H. Malignant otitis externa due to Aspergillus in an immunocompetent patient. Arch Otolaryngol Head Neck Surg 1988;114:554-6

5 Gruver AL, Hudson LL, Sempowski GD. Immunosenescence of ageing. $J$ Pathol 2007;211:144-56

6 Shaw AC, Panda A, Joshi SR, Qian F, Allore HG, Montgomery RR. Dysregulation of human Toll-like receptor function in aging. Ageing Res Rev 2011;10:346-53

7 Frasca D, Diaz A, Romero M, Landin AM, Blomberg BB. Age effects on B cells and humoral immunity in humans. Ageing Res Rev 2011;10:330-5

8 Mani N, Sudhoff H, Rajagopal S, Moffat D, Axon PR. Cranial nerve involvement in malignant external otitis: implications for clinical outcome. Laryngoscope 2007;117:907-10

9 Carfrae MJ, Kesser BW. Malignant otitis externa. Otolaryngol Clin North Am 2008;41:537-49

10 Grandis JR, Curtin HD, Yu VL. Necrotizing (malignant) external otitis: prospective comparison of CT and MR imaging in diagnosis and follow-up. Radiology 1995;196:499-504

11 Strashun AM, Nejatheim M, Goldsmith SJ. Malignant external otitis: early scintigraphic detection. Radiology 1984;150:541-5

12 Handzel O, Halperin D. Necrotizing (malignant) external otitis. Am Fam Physician 2003;68:309-12

13 Stokkel MP, Boot CN, van Eck-Smit BL. SPECT gallium scintigraphy in malignant external otitis: initial staging and followup. Case reports. Laryngoscope 1996;106:338-40

Address for correspondence:

Mr Samit Unadkat,

Department of Otolaryngology,

Southend University Hospital,

Prittlewell Chase,

Westcliff on Sea SS0 0RY, UK

Fax: 02085356834

E-mail: s.unadkat@nhs.net

Mr S Unadkat takes responsibility for the integrity of the content of the paper

Competing interests: None declared 